\title{
Retraction Note: In Vitro Transfection of Plasmid DNA by Amine Derivatives of Gelatin Accompanied with Ultrasound Irradiation
}

Hossein Hosseinkhani' - Teruyoshi Aoyana ${ }^{1,2}$ - Shingo Yamamoto ${ }^{2}$ Osamu Ogawa ${ }^{2}$. Yasuhiko Tabata'

Published online: 3 December 2020

(C) Springer Science+Business Media, LLC, part of Springer Nature 2020

\section{Retraction Note: Pharmaceutical Research volume 19, pages I47 I-I479(2002) https://doi.org/I 0.1023/A: 10204005 I4990}

The Editor-in-Chief has retracted this article [1] at the request of the corresponding author. Figure $1 \mathrm{~B}$ appears to be identical to Figure 1D, despite being under different experimental conditions. The data reported in this article are therefore unreliable. Teruyoshi Aoyama, Shingo Yamamoto, Osamu Ogawa, Yasuhiko Tabata, and Hossein Hosseinkhani agree with this retraction.

[1] Hosseinkhani, H., Aoyama, T., Yamamoto, S. et al. In Vitro Transfection of Plasmid DNA by Amine Derivatives of Gelatin Accompanied with Ultrasound Irradiation. Pharm Res 19, 1471-1479 (2002). https:// doi.org/10.1023/A:1020400514990

The online version of the original article can be found at https://doi.org/l 0 . | 023/A: 10204005 | 4990

Yasuhiko Tabata

yasuhiko@frontier.kyoto-u.ac.jp

Institute for Frontier Medical Sciences, Kyoto University, 53 Kawara-cho Shogoin, Sakyo-ku, Kyoto 606-8507, Japan

2 Department of Urology, Graduate School of Medicine, Kyoto University, 54 Kawara-cho Shogoin Sakyo-ku, Kyoto 606-8507, Japan 